\title{
Modified Adomain Decomposition Method for the Generalized Fifth Order KdV Equations
}

\author{
Huda O. Bakodah \\ Department of Mathematics, Science Faculty for Girls, King Abdulaziz University, Jeddah, KSA \\ Email: h.o.bak@hotmail.com, hbakodah@kau.edu.sa
}

Received September 23, 2012; revised November 6, 2012; accepted November 28, 2012

\begin{abstract}
New modified Adomian decomposition method is proposed for the solution of the generalized fifth-order Korteweg-de Vries (GFKdV) equation. The numerical solutions are compared with the standard Adomian decomposition method and the exact solutions. The results are demonstrated which confirm the efficiency and applicability of the method.
\end{abstract}

Keywords: Modified Adomian Decomposition Method; Fifth Order KdV Equation; Solitary-Wave Solution

\section{Introduction}

The fifth-order (or generalized) $\mathrm{KdV}$ is the essential a model foe several physical phenomena including shallow-water waves near critical value of surface tension and waves in nonlinear LC circuit with mutual inductance between neighbouring inductors [1]. Although no general solution is known, the exact solution of the fifth order $\mathrm{KdV}$ equation has been found for the special case of solitary waves in [2]. In general, the fifth order $\mathrm{KdV}$ need to be solved numerically. Commonly used numerical methods to approximate the solutions of (gfKdV) include finite difference methods, collection methods and Galerkin methods. Kawahara [3] investigated the steady solutions of this equation on the basis of numerical calculations. Boyd [4] and Haupt and Boyd [5] reduced this equation to an ordinary differential equation and then a variety of analyticl and numerical methods are developed. The numerical methodes are based on Newton-Kantorovich pseudo-spectral method and Newton-Kantorovich Galerkin method. In [6] K. Djidjeli et al. proposed finite deference schemes based on a predictor corrector algorithm and a linearized implicit method for the third and fifth order $\mathrm{KdV}$ equations.

However, some of these methods are not easy to use and sometimes require tedious work and calculation [7, 8]. In recent years, Adomian decomposition methods (ADM) [9] have emerged as a powerful tool for a wide class of nonlinear equation [10]. G. Adomian in [11] applied his method to the 5th order KdV equation. In [12, 13], D. Kaya calculated the explicit and numerical solutions of some fifth-order KdV equation by decomposition method and Kaya and El-Sayed in [14] proved the convergence of (ADM) applied to (gfKdV) equation.
A comparative study between (ADM) and Crank Nicholas method presented in [15]. (ADM) has led to several modifications on the method made by various researches in order to improve the accuracy or expansion of the application of the original method. Wazwaz [16] presented a reliable modification of the Adomian decomposition method.

In 2001, Wazwaz presented another type of modification [17] to the (ADM). Wazwaz modifications arises in the initial definition of the operator when applying the (ADM) to the nonlinear equation.

To the best of our knowledge, no attempt is made regarding the solution of generalized fifth order KdV equations by using modified decomposition method. So our main aim in this paper is used the modification ADM to solve the five particular class of the (gfKdV) equations. We generalized an appropriate Adomian's polynomials for (gfKdV) equation will be handle more easily, quickly, and elegantly by implementing the new modified (ADM) rather than traditional methods for the exact solution of which is to be obtained subject to initial condition.

\section{Fifth-Order KdV Equations}

The well-known fifth-order $\mathrm{KdV}$ (fKdV) equations can be shown in the form

$$
u_{t}+a u^{2} u_{x}+b u_{x} u_{x x}+c u u_{x x x}+d u_{x x x x x}=0
$$

where $a, b, c$ and $d$ are nonzeros and real parameters, and $u=u(x, t)$ is a sufficiently smooth function. The (fKdV) is an important mathematical model with wide applications in quantum mechanics and nonlinear optics.

Typical examples widely used in various fields such as solid state physics, plasma physics, fluid physics and 
quantum field theory. A variety of the (fKdV) equations can be developed by changing the real values of the parameters $a, b$ and $c$ [18]. the derivation of these fifthorder forms are derived specific bilinear forms of the so-called Hirota's D-operators. However, five will known forms of the (fKdV) that are of particular interest in the literature. These forms are:

1) The Sawada-Kotera (SK) equation is given by [19]

$$
u_{t}+45 u^{2} u_{x}+15 u_{x} u_{x x}+15 u u_{x x x}+u_{x x x x x}=0
$$

2) The Caudrey-Dodd-Gibbon (CDG) equation is given by [20]

$$
u_{t}+180 u^{2} u_{x}+30 u_{x} u_{x x}+30 u u_{x x x}+u_{x x x x x}=0
$$

3) The Lax equations [21]

$$
u_{t}+30 u^{2} u_{x}+30 u_{x} u_{x x}+10 u u_{x x x}+u_{x x x x x}=0
$$

4) The Kaup-Kuperschmidt (KK) equation $[22,23]$

$$
u_{t}+20 u^{2} u_{x}+25 u_{x} u_{x x}+10 u u_{x x x}+u_{x x x x x}=0
$$

5) The Ito equation [24]

$$
u_{t}+2 u^{2} u_{x}+6 u_{x} u_{x x}+3 u u_{x x x}+u_{x x x x x}=0
$$

\section{The Method of Soultion}

\subsection{Adomian Decomposition Method}

In this section, we give outline and implement Adomian decomposition method for nonlinear equations to obtain analytic and approximate solutions which are obtained in a rapidly convergent series with elegantly computable components by this method. The Adomian approximation series converge quickly. In general, convergence regions of the series are small. Now we outline of the method here in order to obtain the solutions using (ADM), consider the fifth-order KdV Equation (1) in an operator form

$$
L_{t}(u)+a K_{u}+b M_{u}+c N_{u}+d L_{x} u=0,
$$

where the notations $K_{u}=u^{2} u_{x}, M_{u}=u_{x} u_{x x}$, and $N_{u}=u u_{x x x}$. By symbolizing the nonlinear term, respectively. The notation $L_{t}=\frac{\delta}{\delta t}$ and $L_{x}=\frac{\delta^{5}}{\delta x^{5}}$ symbolize the linear differential operators. Assuming the inverse of operator $L_{t}^{-1}$ exists and it can conveniently be taken as the definite integral with respect to $t$ from 0 to $t$, i.e.,

$$
L_{t}^{-1}=\int_{0}^{t}(\cdot) \mathrm{d} t
$$

Thus, applying the inverse operator $L_{t}^{-1}$ to (7) yields;

$$
\begin{aligned}
& L_{t}^{-1} L_{t}(u)=-a L_{t}^{-1}\left(K_{u}\right)-b L_{t}^{-1}\left(M_{u}\right) \\
& -c L_{t}^{-1}\left(N_{u}\right)-d L_{t}^{-1}\left(L_{x} u\right)=0,
\end{aligned}
$$

Therefore, it follows that

$$
\begin{aligned}
& u(x, t)-u(x, 0) \\
& =-a L_{t}^{-1}\left(K_{u}\right)-b L_{t}^{-1}\left(M_{u}\right)-c L_{t}^{-1}\left(N_{u}\right)-d L_{t}^{-1}\left(L_{x} u\right),
\end{aligned}
$$

Since initial value is known and we decompose the unknown function $u(x, t)$ as a sum of components defined by the decomposition series

$$
u(x, t)=\sum_{n=0}^{\infty} u_{n}(x, t),
$$

with $u_{0}$ identified as $u(x, 0)$.

The nonlinear terms $K_{u}, M_{u}$ and $N_{u}$ can be decomposed into infinite series of polynomial given by

$$
\begin{aligned}
& K_{u}=u^{2} u_{x}=\sum_{n=0}^{\infty} A_{n}, \\
& M_{u}=u_{x} u_{x x}=\sum_{n=0}^{\infty} B_{n}, \\
& N_{u}=u u_{x x x x}=\sum_{n=0}^{\infty} C_{n},
\end{aligned}
$$

$A_{n}, B_{n}$, and $C_{n}$ are the so-called Adomian polynomials of $u_{0}, u_{1}, \cdots, u_{n}$. defined by

$$
P_{n}=\frac{1}{n !} \frac{\mathrm{d}^{n}}{\mathrm{~d} \lambda^{n}}\left[\Phi\left(\sum_{k=1}^{\infty} \lambda^{k} u_{k}\right)\right]_{\lambda=0}, n=0,1,2, \cdots .
$$

Substituting (11-14) into (10) gives rise to

$$
\begin{aligned}
& u_{n+1}=-a L_{t}^{-1} A_{n}-b L_{t}^{-1} B_{n}-c L_{t}^{-1} C_{n}-d L_{t}^{-1}\left(L_{x} u_{n}\right), \\
& n \geq 1 .
\end{aligned}
$$

The solution $u(x, t)$ must satisfy the requirements imposed by the initial conditions. Based on the (ADM), we constructed the solution $u(x, t)$ as

$$
\begin{aligned}
& \lim _{n \rightarrow \infty} \varphi_{n}=u(x, t), \\
& \text { where } \varphi_{n}(x, t)=\sum_{k=0}^{n} u_{k}(x, t), n \geq 0 .
\end{aligned}
$$

The decomposition method provides a reliable technique that requires less work if we compared with the traditional techniques.

\subsection{New Modified Adomian Decomposition Method}

In the new modification by Wazwaz [17], we can replace the process of identified $u_{0}$ as $u(x, 0)$ by dividing $u(x, 0)$ by a series of infinite components. We therefore suggest that

$$
u_{0}(x, t)=\sum_{n=0}^{\infty} u_{0}^{n}(x)
$$

A new recursive relationship expressed in the form

$$
u_{0}=u_{0}^{0}
$$




$$
\begin{aligned}
& u_{n+1}=u_{0}^{n+1}-a L_{t}^{-1} A_{n}-b L_{t}^{-1} B_{n}-c L_{t}^{-1} C_{n}-d L_{t}^{-1}\left(L_{x} u_{n}\right), \\
& n \geq 1 .
\end{aligned}
$$

We can observe that algorithm (19) reduces the number of terms involved in each component, and hence the size of calculations is minimized compared to the standard (ADM) only. Moreover this reduction of terms in each component facilitates the construction of Adomian polynomials for nonlinear operators. the new modification overcomes the difficulty of decomposing $u(x, 0)$ and introduces an efficient algorithm that improves the performance of the standard (ADM).

\section{Numerical Experiments}

In this section, we consider some (gfKdV) equations for numerical comparisons based on the new modifications of (ADM). In this paper, we illustrate how the approximate solutions of the (gfKdV) equations are close to exact solutions.

\subsection{Example (1): (Sawada-Kotera Equation)}

we consider the (S-K) Equation [25], with initial condition is given by

$$
u_{0}(x, t)=2 k^{2} \operatorname{sech}^{2}\left(k\left(x-x_{0}\right)\right) .
$$

and the exact solution

$$
u(x, t)=2 k^{2} \operatorname{sech}^{2}\left(k\left(x-16 k^{4} t-x_{0}\right)\right) .
$$

Table 1 shows the difference of the analytical solution and numerical solution of the absolute errors only for 5 iterative.

\subsection{Example (2): (Caudrcy-Dodd-Gbbon (C-D-G) Equation)}

we consider the (C-D-G) equation, with initial condition

$$
u(x, 0)=\frac{k^{2} \mathrm{e}^{k x}}{\left(1+\mathrm{e}^{k x}\right)^{2}} .
$$

and the exact solution

$$
u(x, t)=\frac{k^{2} \mathrm{e}^{\left(k\left(x-k^{4} t\right)\right)}}{\left[1+\mathrm{e}^{\left(k\left(x-k^{4} t\right)\right)}\right]^{2}} .
$$

Table 2 shows the numerical results for example (2) for $k=0.01$.

\subsection{Example (3): (The Lax Equation)}

we consider Lax's fifth order KdV equation with the initial condition:

$$
u(x, 0)=2 k^{2}\left(2-3 \tanh ^{2}\left(k\left(x-x_{0}\right)\right)\right) .
$$

and the exact solution

$$
u(x, t)=2 k^{2}\left(2-3 \tanh ^{2}\left(k\left(x-56 k^{4} t-x_{0}\right)\right)\right) .
$$

Table 3 shows the numerical results for example (3) for $k=0.01, x_{0}=0.0$.

\subsection{Example (4): (Kaup-Kuperschmidt (K-K) Equation)}

We consider the (K-K) equation with the initial condition

$$
u(x, 0)=\frac{24 k^{2} \mathrm{e}^{k x}\left[4 \mathrm{e}^{k x}+\mathrm{e}^{2 k x}+16\right]}{\left[16 \mathrm{e}^{k x}+\mathrm{e}^{2 k x}+16\right]^{2}} .
$$

and the exact solution

$$
u(x, t)=\frac{24 k^{2} \mathrm{e}^{k x-t}\left[4 \mathrm{e}^{k x-k^{5} t}+\mathrm{e}^{2 k x-k^{5} t}+16\right]}{\left[16 \mathrm{e}^{k x-k^{5} t}+\mathrm{e}^{2\left(k x-k^{5} t\right)}+16\right]^{2}} .
$$

Table 4 shows the numerical results for example (4) for $k=0.01$.

\subsection{Example (5): (Ito Equation)}

we consider the Ito equation with the initial condition

$$
u(x, 0)=20 k^{2}-30 k^{2} \operatorname{tanch}^{2}[k x] .
$$

and the exact solution

$$
\begin{aligned}
u(x, t) & =20 k^{2} \\
& -30 k^{2} \operatorname{tanch}^{2}\left[k x-96 k^{4}+x_{0}\right] .
\end{aligned}
$$

Table 5 shows the numerical results for example (5) for $k=0.01$ and $x_{0}=0.0$.

\section{Conclusions and Remarks}

In this work, we proposed new modification of Adomian decomposition method. We solved the five well known forms of the (fKdV) equation with initial conditions. The method has been shown to computationally efficient in these examples that are important to researchers in applied sciences. The obtained results in examples indicted that the new modification of (ADM) was feasible and effective. The method overcomes the difficulties arising in the modified decomposition method established in [16].

The results show that the presented method is powerful mathematical tool for finding good approximate solu- 
Table 1. Absolute error between the exact solution and approximation solution for $\boldsymbol{k}=\mathbf{0 . 0 1}$ and $x_{\mathbf{0}}=\mathbf{0 . 0}$.

\begin{tabular}{ccccccc}
\hline $\mathrm{t} / \mathrm{x}$ & 0.2 & 0.4 & 0.6 & 0.8 & 1.0 & 5.0 \\
\hline 2 & $1.54499 \times 10^{-18}$ & $4.52654 \times 10^{-18}$ & $1.25496 \times 10^{-17}$ & $2.81893 \times 10^{-17}$ & $5.40204 \times 10^{-17}$ & $6.6435 \times 10^{-15}$ \\
4 & $5.36680 \times 10^{-18}$ & $1.12757 \times 10^{-17}$ & $2.7349 \times 10^{-17}$ & $5.87095 \times 10^{-17}$ & $1.10345 \times 10^{-16}$ & $1.32874 \times 10^{-14}$ \\
6 & $1.13841 \times 10^{-17}$ & $2.02746 \times 10^{-17}$ & $4.44252 \times 10^{-17}$ & $9.14253 \times 10^{-17}$ & $1.68892 \times 10^{-16}$ & $1.99336 \times 10^{-14}$ \\
8 & $1.97054 \times 10^{-17}$ & $3.15232 \times 10^{-17}$ & $6.37511 \times 10^{-17}$ & $1.26364 \times 10^{-16}$ & $2.29688 \times 10^{-16}$ & $2.6582 \times 10^{-14}$ \\
10 & $3.02492 \times 10^{-17}$ & $4.50486 \times 10^{-17}$ & $8.52725 \times 10^{-17}$ & $1.63633 \times 10^{-16}$ & $2.92707 \times 10^{-16}$ & $3.32327 \times 10^{-14}$ \\
\hline
\end{tabular}

Table 2. Absolute error between the exact solution and approximation solution for $\boldsymbol{k}=\mathbf{0 . 0 1}$ and $\boldsymbol{x}_{\mathbf{0}}=\mathbf{0 . 0}$.

\begin{tabular}{ccccccc}
\hline $\mathrm{t} / \mathrm{x}$ & 0.2 & 0.4 & 0.6 & 0.8 & 1.0 & 5.0 \\
\hline 2 & $1.96921 \times 10^{-21}$ & $3.11957 \times 10^{-21}$ & $3.63542 \times 10^{-21}$ & $1.7893 \times 10^{-20}$ & $2.62474 \times 10^{-20}$ & $2.48025 \times 10^{-18}$ \\
4 & $3.87141 \times 10^{-21}$ & $2.70138 \times 10^{-21}$ & $9.52285 \times 10^{-21}$ & $2.01336 \times 10^{-20}$ & $5.33583 \times 10^{-20}$ & $5.70681 \times 10^{-18}$ \\
6 & $7.39739 \times 10^{-21}$ & $5.50257 \times 10^{-21}$ & $2.20177 \times 10^{-20}$ & $3.91461 \times 10^{-20}$ & $7.69124 \times 10^{-20}$ & $8.93996 \times 10^{-18}$ \\
8 & $1.89429 \times 10^{-21}$ & $4.74685 \times 10^{-21}$ & $2.75676 \times 10^{-20}$ & $5.79897 \times 10^{-20}$ & $9.69092 \times 10^{-20}$ & $1.21797 \times 10^{-17}$ \\
10 & $6.82819 \times 10^{-21}$ & $7.21049 \times 10^{-21}$ & $2.61724 \times 10^{-20}$ & $5.63358 \times 10^{-20}$ & $1.20126 \times 10^{-19}$ & $1.54092 \times 10^{-17}$ \\
\hline
\end{tabular}

Table 3. The exact and approximation solution of Lax equation for $k=0.01$.

\begin{tabular}{ccccccc}
\hline $\mathrm{t} / \mathrm{x}$ & 0.2 & 0.4 & 0.6 & 0.8 & 1.0 & 5.0 \\
\hline 2 & $5.76197 \times 10^{-14}$ & $1.15211 \times 10^{-13}$ & $1.72788 \times 10^{-13}$ & $2.30342 \times 10^{-13}$ & $2.87867 \times 10^{-13}$ & $1.42104 \times 10^{-12}$ \\
4 & $1.15281 \times 10^{-13}$ & $2.30464 \times 10^{-13}$ & $3.45618 \times 10^{-13}$ & $4.60727 \times 10^{-13}$ & $5.75777 \times 10^{-13}$ & $2.84213 \times 10^{-12}$ \\
6 & $1.72985 \times 10^{-13}$ & $3.45759 \times 10^{-13}$ & $5.18489 \times 10^{-13}$ & $6.91153 \times 10^{-13}$ & $8.63729 \times 10^{-13}$ & $4.26326 \times 10^{-12}$ \\
8 & $2.3073 \times 10^{-13}$ & $4.61096 \times 10^{-13}$ & $6.91403 \times 10^{-13}$ & $9.21621 \times 10^{-13}$ & $1.15172 \times 10^{-12}$ & $5.68444 \times 10^{-12}$ \\
10 & $2.88518 \times 10^{-13}$ & $5.76475 \times 10^{-13}$ & $8.64358 \times 10^{-13}$ & $1.15213 \times 10^{-12}$ & $1.43976 \times 10^{-12}$ & $7.10566 \times 10^{-12}$ \\
\hline
\end{tabular}

Table 4. Absolute error between the exact solution and approximation solution for $\boldsymbol{k}=\mathbf{0 . 0 1}$ and $x_{0}=\mathbf{0 . 0}$.

\begin{tabular}{ccccccc}
\hline $\mathrm{t} / \mathrm{x}$ & 0.2 & 0.4 & 0.6 & 0.8 & 1.0 & 5.0 \\
\hline 2 & $1.46896 \times 10^{-16}$ & $7.86155 \times 10^{-17}$ & $1.0355 \times 10^{-17}$ & $5.77728 \times 10^{-17}$ & $1.25763 \times 10^{-16}$ & $2.24215 \times 10^{-15}$ \\
4 & $2.9378 \times 10^{-16}$ & $1.57193 \times 10^{-16}$ & $2.07239 \times 10^{-17}$ & $1.15638 \times 10^{-16}$ & $2.51752 \times 10^{-16}$ & $7.63093 \times 10^{-16}$ \\
6 & $4.40678 \times 10^{-16}$ & $2.35791 \times 10^{-16}$ & $3.10725 \times 10^{-17}$ & $1.73475 \times 10^{-16}$ & $3.77733 \times 10^{-16}$ & $7.15916 \times 10^{-16}$ \\
8 & $5.87582 \times 10^{-16}$ & $3.14383 \times 10^{-16}$ & $4.14144 \times 10^{-17}$ & $2.31306 \times 10^{-16}$ & $5.03728 \times 10^{-16}$ & $2.19495 \times 10^{-15}$ \\
10 & $7.34466 \times 10^{-16}$ & $3.92994 \times 10^{-16}$ & $5.17698 \times 10^{-17}$ & $2.89164 \times 10^{-16}$ & $6.29737 \times 10^{-16}$ & $3.67398 \times 10^{-15}$ \\
\hline
\end{tabular}

Table 5. Absolute error between the exact solution and approximation solution for $\boldsymbol{k}=\mathbf{0 . 0 1}$ and $\boldsymbol{x}_{\mathbf{0}}=\mathbf{0 . 0}$.

\begin{tabular}{ccccccc}
\hline $\mathrm{t} / \mathrm{x}$ & 0.2 & 0.4 & 0.6 & 0.8 & 1.0 & 5.0 \\
\hline 2 & $1.40995 \times 10^{-16}$ & $1.41060 \times 10^{-16}$ & $1.41178 \times 10^{-16}$ & $1.4137 \times 10^{-16}$ & $1.4168 \times 10^{-16}$ & $7.17504 \times 10^{-16}$ \\
4 & $5.63981 \times 10^{-16}$ & $5.64238 \times 10^{-16}$ & $5.64685 \times 10^{-16}$ & $5.65365 \times 10^{-16}$ & $5.66365 \times 10^{-16}$ & $1.74347 \times 10^{-15}$ \\
6 & $1.26896 \times 10^{-15}$ & $1.26953 \times 10^{-15}$ & $1.27052 \times 10^{-15}$ & $1.27199 \times 10^{-15}$ & $1.27405 \times 10^{-15}$ & $3.07794 \times 10^{-15}$ \\
8 & $2.25592 \times 10^{-15}$ & $2.25695 \times 10^{-15}$ & $2.25868 \times 10^{-15}$ & $2.26123 \times 10^{-15}$ & $2.26475 \times 10^{-15}$ & $4.7209 \times 10^{-15}$ \\
10 & $3.52488 \times 10^{-15}$ & $3.52648 \times 10^{-15}$ & $3.52918 \times 10^{-15}$ & $3.5331 \times 10^{-15}$ & $3.53845 \times 10^{-15}$ & $6.67236 \times 10^{-15}$ \\
\hline
\end{tabular}




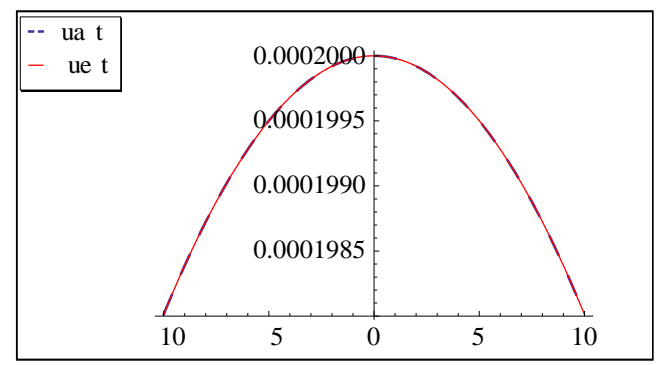

Figure 1. The exact and the approximation solution of $s-k$ equation for $t=10$ and $k=0.01$.

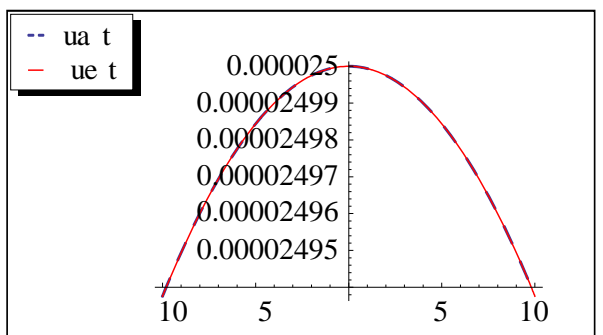

Figure 2. The exact and the approximation solution of C-D-G equation for $t=10$ and $k=0.01$.

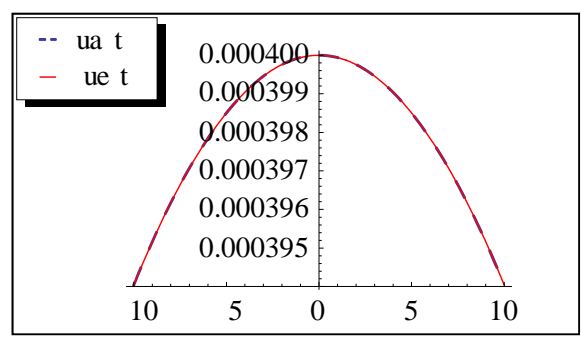

Figure 3. The exact and the approximation solution of Lax equation for $t=10$ and $k=0.01$.

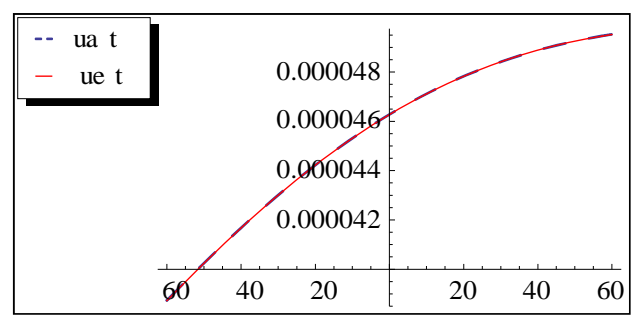

Figure 4. The exact and the approximation solution of K-K equation for $t=10$ and $k=0.01$.

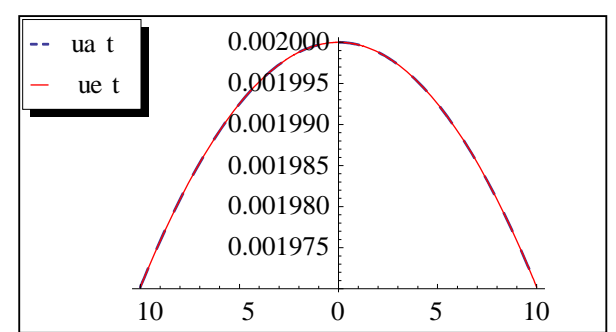

Figure 5. The exact and the approximation solution of Ito equation for $t=10$ and $k=0.01$. tions of (gfKdV) equation with initial conditions and results are found to be in good agreement with the exact solution as shown from Figures 1-5. In addition, no linearization or perturbation is required by the method.

\section{REFERENCES}

[1] H. Nagashima, "Experiment on Solitary Waves in the Nonlinear Transmission Line Discribed by the Equation $u_{t}+u u_{\xi}-u_{\xi \xi \xi \xi \xi}=0$," Journal of the Physical Society of Japan, Vol. 47, 1979, pp. 1387-1388. doi:10.1143/JPSJ.47.1387

[2] Y. Yamaoto and E. Takizawa, "On a Solution on Non-Linear Time-Evolution Equation of Fifth-Order," Journal of the Physical Society of Japan, Vol. 50, 1981, pp. 1421-1422. doi:10.1143/JPSJ.50.1421

[3] T. Kawahrara, "Oscillatary Solitary Waves in Dispersive Media,” Journal of the Physical Society of Japan, Vol. 33, 1972, pp. 260-264. doi:10.1143/JPSJ.33.260

[4] J. P. Boyd, "Solitons from Sine Waves: Analytical and Numerical Methods for Non-Integrable Solitary and Conoidal Waves,” Physica 21D, 1986, pp. 227-246.

[5] S. E. Haupt and J. P. Boyd, "Modelling Nonlinear Resonance: A Modification to the Stokes Perturbation Expansion,” Wave Motion, Vol. 10, No. 1, 1988, pp. 83-98.

[6] K. Djidjeli, W. G. Price, E. H. Twizell and Y. Wang, "Numerical Methods for the Soltution of the Third and Fifth-Order Disprsive Korteweg-De Vries Equations," Journal of Computational and Applied Mathematics, Vol. 58, No. 3, 1995, pp. 307-336. doi:10.1016/0377-0427(94)00005-L

[7] A. M. Wazwaz, "Analytic Study on the Generalized KdV Equation: New Solution and Periodic Solutions,” Elsevier, Amsterdam, 2006.

[8] M. T. Darvishi and F. Khani, "Numerical and Explicit Solutions of the Fifth Order Korteweg-De Vries Equations,” Elsevier, Amsterdam, 2007.

[9] G. Adomian, "Solving Frontier Problems of Physics: The Decomposition Method,” Kluwer Academic Publisher, Dordrecht, 1994.

[10] D. Kaya, "The Use of Adomian Decomposition Methods for Solving a Specific Nonlinear Partial Differential Equations," Bulletin of the Belgian Mathematical Society Simon Stevin, Vol. 9, No. 3, 2002, pp. 343-349.

[11] G. Adomian, "The Fifth-Order Korteweg-De Vries Equation," International Journal of Mathematics and Mathematical Sciences, Vol. 19, No. 2, 1996, p. 415. doi:10.1155/S0161171296000592

[12] D. Kaya, "An Explicit and Numerical Solutions of Some Fifth-Order KdV Equation by Decomposition Method," Applied Mathematics and Computation, Vol. 144, No. 2-3, 2003, pp. 353-363. doi:10.1016/S0096-3003(02)00412-5

[13] D. Kaya, "An Application for the Higher Order Modified KdV Equation by Decomposition Method," Communications in Nonlinear Science and Numerical Simulation, Vol. 10, No. 6, 2005, pp. 693-702. doi:10.1016/j.cnsns.2003.12.009 
[14] D. Kaya and S. M. El-Sayed, "On a Generalized Fifth Order KdV Equations,” Physics Letters A, Vol. 310, No. 1, 2003, pp. 44-51. doi:10.1016/S0375-9601(03)00215-9

[15] M. A. Helal and M. S. Mehanna, “A Comparative Study between Two Different Methods for Solving the General Korteweg-De Vries Equation (GKDV)," Chaos, Solitons \& Fractals, Vol. 33, No. 3, 2007, pp. 725-739. doi:10.1016/j.chaos.2006.11.011

[16] A. M. Wazwaz, "A Reliable Technique for Solving Linear and Nonlinear Schrodinger Equations by Adomian Decompostion Method," Bulletin of the Institute of Mathematics, Vol. 29, No. 2, 2001, pp. 125-134.

[17] A. M. Wazwaz and S. M. El-Sayed, "A New Modification of the Adomian Decoomposition Method for Linear and Nonlinear Operators," Applied Mathematics and Computations, Vol. 122, 2001, pp. 393-405.

[18] A. M. Wazwaz, "Partial Differential Equations and Solitary Waves Theory," Higher Education Press, Beijing; Spinger-Verlag, Berlin, 2009.

[19] K. Sawada and T. Kotera, "A Method for Finding N-Soliton Solutions of the KdV. Equation and the KbVLike Equation,” Progress of Theoretical Physics, Vol. 51, No. 5, 1974, pp. 1355-1367. doi:10.1143/PTP.51.1355
[20] P. J. Coudery, R. K. Dodd and J. D. Gibbon, “A New Hierarchy of Korteweg-De Vries Equations,” Proceedings of the Royal Society A, Vol. 351, No. 1666, 1976, pp. 407-422. doi:10.1098/rspa.1976.0149

[21] P. D. Lax, "Integrals of Nonlinear Equations of Evolution and Solitary Waves," Communications on Pure and Applied Mathematics, Vol. 21, No. 5, 1968, pp. 467-490. doi:10.1002/cpa.3160210503

[22] D. J. Kaup, “On the Iverse Scattering Problem for Cubic Eigenvalue Problems of the Class $\psi_{x x x}+6 Q \psi_{x}+6 R \psi=\lambda \psi$," Studies in Applied Mathematics, Vol. 62, 1980, pp. 189-216.

[23] B. A. Kuperschmidt, "A Super KdV Equation: An Integral System,” Physics Letters A, Vol. 102, No. 5-6, 1984, pp. 213-215. doi:10.1016/0375-9601(84)90693-5

[24] M. Ito, "An Extension of Nonlinear Evolution Equations of the KdV (mKdV) Type of Higher Orders," Journal of the Physical Society of Japan, Vol. 49, No. 2, 1980, pp. 771-778. doi:10.1143/JPSJ.49.771

[25] Y. Lei, Z. Fajiang and W. Yinghai, “The Homogeneous Balance Method, Lax Pair Hirota Transformation and a General Fifth Order KdV Equation,” Chaos Solutions Fractals, Vol. 13, 2002, p. 337. 